\title{
RECOLONISATION D'UN COURS D'EAU CORSE DÉVASTÉ : CONTRIBUTION RESPECTIVE DES TRUITES SAUVAGES ET DOMESTIQUES.
}

\author{
D. BEAUDOU (1), D. BARIL (1), B. ROCHÉ (2), M. LE BARON (1), \\ G. CATTANEO-BERREBI (3), P. BERREBI (3)
}

(1) Conseil Supérieur de la Pêche, Délégation Régionale $n^{\circ} 8,55$ chemin du Mas de Matour, 34790 GRABELS, France.

(2) Direction Régionale de l'Environnement de Corse, S.E.M.A., BP 605, 20601 BASTIA Cedex, France.

(3) Laboratoire Génome et Populations, Université Montpellier II, CC063, place Eugène Bataillon, 34095 MONTPELLIER Cedex 05, France.

\section{RÉSUMÉ}

L'Abatesco, petite rivière côtière au centre-est de la Corse, a subi en septembre 1989 une crue dévastatrice. Dans le lit principal, la perturbation de l'habitat et de la végétation rivulaire a entraîné une destruction quasi totale de la faune benthique et des populations de truites communes (Salmo trutta L.). Près de 20000 truitelles ( 5 centimètres de longueur en moyenne) d'origine domestique ont été déversées au printemps 1990 dans le cours principal et dans ses affluents. Les années suivantes, seule la tête du bassin a été repeuplée. D'autre part, la pêche a été interdite jusqu'en mars 1993. Deux sites de l'Abatesco ont été prospectés à plusieurs reprises entre septembre 1989 et septembre 1993 afin d'évaluer les populations de truites. En juin 1993, 30 truites ont été prélevées dans l'Abatesco pour analyse génétique (allozymes).

La recolonisation des différentes stations nécessite au moins 2 années. Bien que l'influence exacte des repeuplements soit difficilement quantifiable, on observe que la reconstitution de la population salmonicole s'effectue principalement à partir des populations des affluents qui ont mieux résisté à la crue. L'analyse génétique montre qu'en juin 1993, $92 \%$ des truites étaient d'origine sauvage (allèle enzymatique $L D H-5^{*} 105$ ) et que $72 \%$ appartenaient à la forme endémique corse (allèle $\mathrm{LDH}-3^{*} 40$ ), que l'on trouve plus généralement en haute altitude.

Ainsi, le suivi de ce phénomène naturel a montré la vitalité de la reconstitution de la population à partir de truites sauvages.

Mots-clés : crues, habitat, recolonisation, truite, Corse.

\section{RECOLONIZATION IN A DEVASTATED CORSICAN RIVER : RESPECTIVE CONTRIBUTION OF WILD AND DOMESTIC BROWN TROUT.}

\begin{abstract}
The present study was carried out in the Abatesco River basin on the Eastern coast of Corsica. In September 1989, this small river basin was devastated by a storm spate. In the main river bed, the destruction of the riparian vegetation and the perturbation of the habitat led to a nearly complete loss of the benthic fauna and thus of the brown trout (Salmo trutta L.) populations. About 20000 domestic fingerlings $(5 \mathrm{~cm}$ mean length) were stocked during the spring of 1990 in the main river and its tributaries. In subsequent years, stocking practices were limited to the head of the basin. Fishing was prohibited until March
\end{abstract}


1993. Two sites were sampled five times from September 1989 to September 1993 for the evaluation of trout densities. In June 1993, 30 individuals were sampled in the Abatesco to investigate allozyme polymorphism.

Recolonization required at least two years. Although the exact influence of stocking practices was difficult to evaluate, we found that brown trout restoration was mainly due to the populations of the tributaries, which had been less disturbed by the spate. The genetic analyses showed that, in June $1993,92 \%$ of the trout carried the wild allele $\mathrm{LDH}-5^{\star} 105$ and $72 \%$ the endemic Corsican allele $\mathrm{LDH}-3^{*} 40$, which is generally only found in basin head populations.

Thus, this study has shown that the wild population was primarily restored by the surviving individuals, particularly those from the tributaries that escaped the spate.

Key-words : spate, habitat, recolonization, trout, Corsica.

\section{INTRODUCTION}

Alors que l'impact des repeuplements en truite commune (Salmo trutta L.) sur les populations en place est largement discuté (GUYOMARD, 1989a), nous pouvons nous interroger sur leur efficacité lorsque la population naturelle est réduite. La rivière côtière Abatesco, située au centre-est de la Corse (Haute-Corse), nous fournit un bon modèle pour étudier les capacités de recolonisation d'une population de truites fortement affectée par une crue naturelle.

Le 1 er septembre 1989, le bassin de l'Abatesco, ainsi que les deux bassins les plus proches du Travo et du Fium'Orbo, ont été dévastés par un épisode pluvieux très important, d'une période de retour de 40 ans. Le peuplement salmonicole de l'Abatesco a été laminé par la violence de la crue sous l'effet cumulé des vitesses de courant et du transport solide (sable, blocs). En l'absence de station de jaugeage, le débit maximum a été estimé à $550 \mathrm{~m}^{3} / \mathrm{s}$ (D.D.E., 1990a, 1990b et 1994), alors que le module peut être évalué à environ $2 \mathrm{~m}^{3} / \mathrm{s}$ et le débit moyen mensuel sec de récurrence de 5 ans autour de $190 \mathrm{l} / \mathrm{s}$ (DIREN Corse, SEMA, 1993). On observe une quasi-disparition des populations salmonicoles liée à la violence de la crue, alors que pour les populations d'anguilles l'impact semble plus limité.

Le protocole d'étude consiste, après ce constat de quasi-disparition des truites sur le cours de l'Abatesco, à suivre la dynamique de recolonisation de cette rivière (BARIL et LE BARON, 1993). Afin de soutenir ce processus, les gestionnaires ont procédé à la mise en résenve totale du bassin de l'Abatesco pendant trois ans (c'est-à-dire jusqu'en mars 1993) et ont effectué des réempoissonnements. Un premier déversement de 20000 truitelles d'origine domestique $(4-6 \mathrm{~cm})$ a eu lieu au printemps 1990 sur tout le cours de l'Abatesco et sur les affluents, suivi de repeuplements réguliers sur l'amont du bassin les années suivantes.

La dynamique de recolonisation de ce cours d'eau a été analysée d'une part grâce à des inventaires par pêche électrique réalisés sur deux stations immédiatement après la crue (septembre 1989), après un an (octobre 1990), deux ans (septembre 1991) et quatre ans (octobre 1993). Un échantillonnage supplémentaire a eu lieu en février 1993 avant la réouverture de la pêche. D'autre part, en juin 1993, 30 truites ont été prélevées pour analyse génétique par électrophorèse des protéines enzymatiques.

Les cours d'eau Corse présentent une zonation piscicole simple. Le peuplement piscicole est réduit à trois espèces : la truite commune (Salmo trutta), l'anguille (Anguilla anguilla) et la blennie (Blennius fluviatilis) (ROCHÉ, 1987). Leur distribution est conditionnée principalement par un gradient d'altitude. Sur la partie inférieure des cours d'eau (0 à $200 \mathrm{~m}$ d'altitude), l'anguille domine numériquement (zone à anguille). La blennie se maintient dans cette zone. Au-dessus de $200 \mathrm{~m}$ d'altitude, la truite domine. Elle est accompagnée d'anguilles jusqu'à environ $800 \mathrm{~m}$ (zone à truite inférieure). Au-dessus de cette altitude, seule la truite demeure (zone à truite supérieure).

Comme sur la plus grande partie du bassin méditerranéen français, nous disposons en Corse de marqueurs électrophorétiques permettant de différencier les truites sauvages des individus de pisciculture, notamment la lactate déshydrogénase $5\left(\mathrm{LDH}-5^{*}\right)$ de l'oeil. 
GUYOMARD (1989b) a mis en évidence, sur deux échantillons du versant est et deux du versant ouest, que les populations corses sont fixées pour l'allèle 105, comme les populations méditerranéennes continentales. Les souches de pisciculture, utilisées en Corse pour les repeuplements, proviennent du continent et sont porteuses de l'allèle 100 . Bien que d'autres locus soient informatifs (GUYOMARD et KRIEG, 1986), nous nous sommes, dans un premier temps, limités à l'analyse des locus de l'oeil. Au locus $L D H-5^{*}$, déjà utilișé sur différents bassins pour évaluer l'effet des repeuplements, s'ajoute le locus $L D H-3^{*}$. II présente, en effet, un allèle caractéristique des populations corses ancestrales, $L D H-3^{*} 40$ (BERREBI, 1994), probablement déjà observé sous la dénomination $L D H-3^{*} 65$ par GUYOMARD et KRIEG (1986).

\section{MATÉRIEL ET MÉTHODES}

\subsection{Le cours d'eau}

L'Abatesco est une petite rivière côtière $(40 \mathrm{~km}$ de longueur) située en Haute-Corse. Elle présente un bassin versant de $89 \mathrm{~km}^{2}$. La qualité des eaux est excellente sur tout son cours (classe de qualité $A$, selon le Schéma de Vocation Piscicole, FÉDÉRATION DES AAPP, 1991).

\subsection{Etude des populations de truites}

Les deux stations d'étude ont été choisies de façon à prospecter les deux grandes zones piscicoles:

- la station $n^{\circ} 1$, au lieu-dit Catastaju, est située en zone à truite inférieure à une altitude de $500 \mathrm{~m}$. Les anguilles représentent moins de $5 \%$ des effectifs capturés lors des différents inventaires.

- la station $\mathrm{n}^{\circ} 2$ est placée face au village de Piétrapola, à une altitude de $180 \mathrm{~m}$, dans la partie supérieure de la zone à anguilles. Celles-ci constituent en effet 30 à $98 \%$ des effectifs capturés sur cette station.

Les inventaires des peuplements piscicoles sont réalisés par pêche électrique par prospection complète, cette technique étant bien adaptée aux petits cours d'eau de faible profondeur $(<1 \mathrm{~m})$ dans lesquels il est possible de progresser à pied. Ces inventaires sont réalisés :

- soit par deux passages successifs, avec retrait des individus pêchés ; les estimations sont alors réalisées par la méthode de CARLE et STRUB (1978) (pêches de septembre 1991, février 1993 et octobre 1993) ;

- soit, par un seul passage (pêches de septembre 1989 et octobre 1990, pour la station 1). Le peuplement total est alors estimé en utilisant les efficacités moyennes de pêche du premier passage observées en septembre 1991 et octobre $1993: 70 \%$ pour les truites de moins de $20 \mathrm{~cm}$ et $65 \%$ pour celles de longueur totale $\geq 20 \mathrm{~cm}$.

A l'exception d'un sondage réalisé sur $50 \mathrm{~m}$ le 12 septembre 1989 sur la station n०1 (Catastaju) compte tenu du très faible effectif de poissons présent juste après la crue, les longueurs échantillonnées varient entre 92 et $140 \mathrm{~m}$ pour la station $n^{\circ} 1$ (Catastaju) et 100 et $111 \mathrm{~m}$ pour la station $\mathrm{n}^{\circ} 2$ (Piétrapola). Afin de standardiser les résultats, les histogrammes de fréquence de taille sont donc présentés par $100 \mathrm{~m}$ de rive. Afin d'individualiser les cohortes, des déterminations d'âge ont été effectuées sur des prélèvements d'écailles réalisés en septembre 1991 sur un ensemble de 25 individus issus des différentes stations échantillonnées.

\subsection{Etude génétique}

Le 4 juin 1993, 30 truites ont été prélevées à la station $n^{\circ} 2$ (Piétrapola) et disséquées sur place (longueur totale comprise entre $110 \mathrm{~mm}$ et $230 \mathrm{~mm}$, soit des individus d'age $1+$ à $3+$ ). Les organes sont immédiatement congelés dans de l'azote liquide. Les 
techniques d'électrophorèse sur gels d'amidon horizontaux sont celles décrites par PASTEUR et al. (1987), avec les tampons et les solutions de coloration de KRIEG (1984). Deux systèmes enzymatiques présents dans l'oeil ont été étudiés : créatine kinase CK (E.C. 2.7.3.2) et L-lactate déshydrogénase LDH (E.C.1.1.1.27). Sur les 5 locus analysés $\left(C K-3^{*}, L D H-1^{*}, L D H-3^{*}, L D H-4^{\star}\right.$ et $\left.L D H-5^{*}\right)$, seuls les locus $L D H-3^{*}$ et $L D H-5^{\star}$ sont polymorphes et seront décrits ici.

\section{RÉSULTATS ET DISCUSSION}

\subsection{Avant-propos sur la dynamique des populations de truites}

Comme dans d'autres secteurs géographiques (par exemple le Scorff, MAISSE et BAGLINIĖRE (1991)), ROCHÉ (1987) souligne que le fonctionnement des populations de truites ne peut être appréhendé qu'à l'échelle du bassin versant, notamment en ce qui concerne le recrutement. En effet, les affluents et les sous-affluents, offrant des substrats plus favorables, jouent un rôle important dans la reproduction de l'espèce. L'axe principal présente généralement peu de biotopes favorables au frai, en raison de brusques variations de débit et de la violence des crues qui remanient fréquemment la granulométrie des fonds. Le frai a donc lieu essentiellement dans la zone à truite supérieure et sur les affluents. Les échanges entre les différentes zones se réalisent grâce à une double migration : la dévalaison des alevins de l'année à partir du mois d'août, suivie au mois d'octobre par la remontée des géniteurs sur les zones de frayères. En zone à truite inférieure, le recrutement s'effectue sur la classe d'âge $0+$ à partir de la zone à truite supérieure et des affluents. Par contre, les truitelles de un an vont elles-mêmes quitter cette zone pour se déplacer vers l'aval. Sur la zone à anguille, le recrutement s'effectue par conséquent essentiellement par les $1+$ provenant de l'amont et d'une façon moindre par les $0+$, issus des affluents.

\subsection{Etude des populations de truites de l'Abatesco}

Comme nous l'avons souligné dans l'introduction, la population de truites est très réduite sur le cours d'eau principal après la crue. Les sondages, effectués le 12 septembre 1989 , ont permis de capturer respectivement 5 truites sur $50 \mathrm{~m}$ sur la station $n^{\circ} 1$ (Catastaju), et une truite sur $100 \mathrm{~m}$ de longueur de rive sur la station $\mathrm{n}^{\circ} 2$ (Piétrapola), située à l'aval et encore plus touchée (tableau I).

Tableau I : Estimation du nombre de truites pour $100 \mathrm{~m}^{2}$ de rivière (densité = Dens.) et de la masse de truites en $\mathrm{kg}$ pour $100 \mathrm{~m}^{2}$ (biomasse = Biom.). Les estimations de ces paramètres en 1989 et 1990 sont obtenues avec un seul passage en extrapolant les valeurs d'efficacité de pêche observées en 1991 et 1993 (deux passages).

Table I : Estimated numbers (Dens. = density) and biomass (Biom. = biomass, in $\mathrm{kg}$ ) of trout per $100 \mathrm{~m}^{2}$. The 1989 and 1990 values (which were obtained using only one passage upstream) were adjusted using the catch efficiency calculated for 1991 and 1993 samples (two passages) using the method of CARLE and STRUB (1978).

\begin{tabular}{lcccccccccc}
\hline & \multicolumn{2}{c}{$12 / 09 / 89$} & \multicolumn{2}{c}{$9 / 10 / 90$} & \multicolumn{2}{c}{$4 / 09 / 91$} & \multicolumn{2}{c}{$17 / 02 / 93$} & \multicolumn{2}{c}{$21 / 09 / 93$} \\
\hline Station & Dens. & Biom. & Dens. & Biom. & Dens. & Biom. & Dens. & Biom. & Dens. & Biom. \\
\hline$n^{\circ} 1$ - Catastaju & 4 & 0,3 & 97 & 1,8 & 63 & 2,0 & 41 & 2,0 & 37 & 1,2 \\
\hline$n^{\circ} 2$ - Piétrapola & 0,3 & 0,01 & & & 26 & 1,0 & 23 & 0,8 & 12 & 0,5 \\
\hline
\end{tabular}


L'évolution des populations présente une densité maximale en octobre 1990 (97 ind/100 $\mathrm{m}^{2}$ sur la station $\mathrm{n}^{\circ} 1$ ), densité qui décroît ensuite. Ce pic semble lié à une artificialisation des densités, conséquence du déversement massif d'alevins sur tout le bassin. Sur la station $n^{\circ} 1$, l'étalement de la cohorte de juvéniles de l'année $(0+)$ (figure 1) et des disparités morphologiques entre les alevins laissent supposer que cette cohorte est composée à la fois de juvéniles sauvages et de juvéniles domestiques, dont les tailles plus importantes seraient liées à une éclosion plus précoce en pisciculture. Une régulation s'opère ensuite en fonction de la capacité biogénique du milieu. Les biomasses se stabilisent autour de $2 \mathrm{~kg} / 100 \mathrm{~m}^{2}$ sur la station $\mathrm{n}^{\circ} 1$, avec un maximum en septembre 1991 .

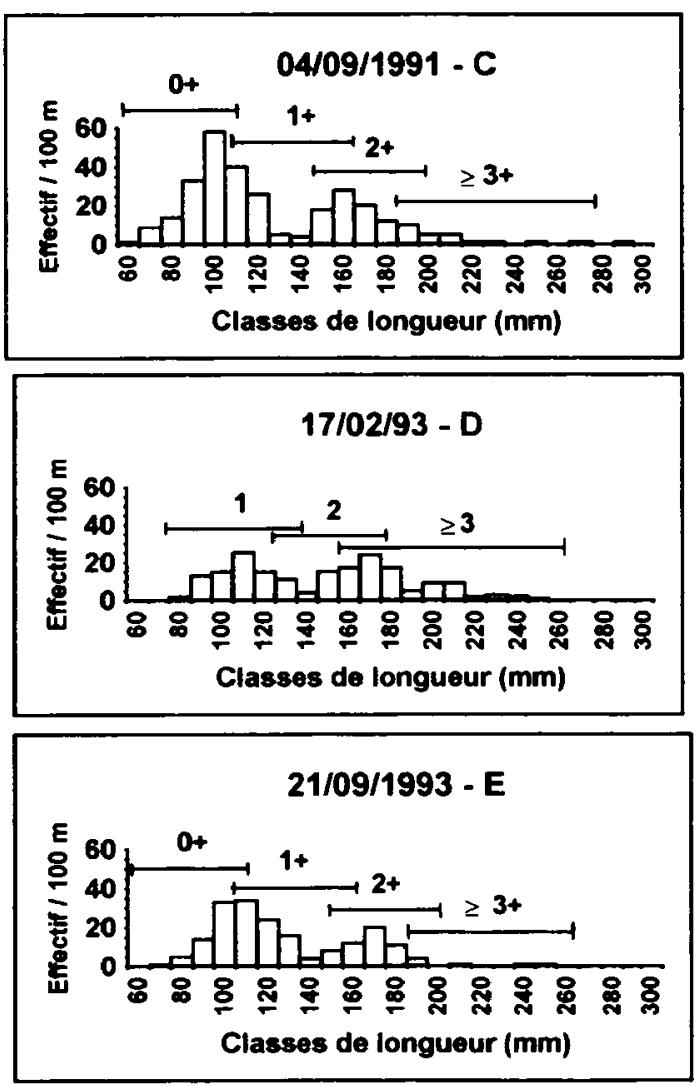

Figure 1 : Effectif capturé par classe de taille sur la station $n^{\circ} 1$ (Catastaju) : $A=$ 12 jours après la crue, $B=$ environ 6 mois après le déversement massif du printemps 1990, $C$ = environ 6 mois après le déversement en tête de bassin, $D=$ avant la réouverture de la pêche, $E=6$ mois après la réouverture de la pêche. Les âges sont déduits d'une relation longueurâge obtenue par l'analyse des écailles de 25 individus de taille variée capturés en septembre 1991.

Figure 1 : Numbers of trout sampled in each length class in station $n^{\circ} 1$ (Catastaju) : $A=12$ days after the spate, $B=$ about 6 months after heavy stocking in the spring of 1990, C = about 6 months after upstream stocking, $D=$ just before the angling season, $E=6$ months after the beginning of the angling season. Age classes were determined from a length-age relationship established by the study of the scales of 25 trout caught in September 1991. 
Pour la station $n^{\circ} 1$ (Catastaju), la réduction sensible de la biomasse entre le mois de février et de septembre 1993 (respectivement 2,0 puis $1,2 \mathrm{~kg} / 100 \mathrm{~m}^{2}$ ) peut être essentiellement imputée à la réouverture de la pêche au mois de mars 1993. En effet, le nombre d'individus de longueur $\geq 18 \mathrm{~cm}$ diminue de $62 \%$ entre février et septembre (figure 1 ). Pour la station $n^{\circ} 2$ (Piétrapola), la baisse touche toutes les classes de tailles et pourrait être attribuée à la pêche et au braconnage sur les petits individus.

Ne disposant pas de données antérieures à la crue sur ce bassin, il est difficile d'évaluer la vitesse de retour aux densités et aux biomasses présentes avant la perturbation. Cependant, les différentes valeurs observées s'inscrivent dans les gammes de variations observées par ROCHÉ (1987) sur des stations situées en zone à truite inférieure (200 à $500 \mathrm{~m}$ d'altitude).

Afin de comprendre les mécanismes de recolonisation, les différentes cohortes, identifiées grâce aux déterminations d'âge réalisés sur les écailles sont positionnées sur les histogrammes (figure 1). Pour ce faire, les déterminations d'âge réalisés sur les écailles prélevées en septembre 1991 ont été extrapolées aux autres dates de pêche. On observe que dès 1991, sur les deux stations, la population est composée d'au moins quatre classes d'âge distinctes $(0+$ à $3+)$. Les truites $2+$ sont nées avant la crue de septembre 1989. Ceci permet de supposer que des juvéniles de l'année $(0+)$ aient survécu à la crue sur les parties amont du bassin et aient ensuite colonisé cette zone par dévalaison. On observe, en effet, la présence de poissons de 1 an sur la station de Catastaju en octobre 1990, alors que sur le cours d'eau principal, seules des truites d'une taille supérieure à $15 \mathrm{~cm}$ ont été capturées.

Sur les différents histogrammes, on observe une cohorte de $1+$ réduite, alors que les cohortes de $0+$ et de $2+$ sont relativement importantes. Ce déficit est caractéristique des populations de la zone à truite inférieure, puisqu'à cette période de l'année, les truitelles de un an, présentes sur la station après l'hiver, se sont déplacées vers l'aval (ROCHÉ, 1987).

\subsection{Etude génétique}

Les résultats de l'étude génétique (tableau II) montrent que la population présente une forte fréquence $(92 \%)$ de l'allèle méditerranéen $L D H-5^{\star} 105$ et également une forte fréquence $(72 \%)$ de l'allèle caractéristique des populations corses endémiques : $L D H-3^{*} 40$.

Tableau II : Fréquences alléliques de l'échantillon de 30 truites prélevé en juin 1993.

Table II : Allelic frequencies of the June 1993 sample (30 trout).

\begin{tabular}{ccccc}
\hline & \multicolumn{2}{c}{$L D H-3^{*}$} & \multicolumn{2}{c}{$L D H-5^{\star}$} \\
\hline Allèle & 40 & 100 & 100 & 105 \\
\hline Fréquence & 0,72 & 0,28 & 0,08 & 0,92 \\
\hline
\end{tabular}

Aucun individu prélevé ne présente le génotype des individus domestiques ( $L D H-3^{*} 100 / 100$ et $\left.L D H-5^{*} 100 / 100\right)$. L'introgression par des gènes domestiques ( $8 \%$ de $L D H-5^{\star} 100$ ) est donc liée aux repeuplements antérieurs à la crue ayant donné lieu à des croisements avec la souche sauvage.

L'effet du déversement de truitelles du printemps 1990 ne peut pas être évalué directement par cet échantillon. En effet, ces truites appartiennent à la cohorte $3+$ en juin 1993, cohorte peu échantillonnée dans ce prélèvement (6 individus $\geq 18 \mathrm{~cm}$ ). Cependant, on 
peut dire que ces 6 individus, potentiellement issus de la souche sauvage ou du déversement du printemps 1990, indiquent que l'effet final de l'apport artificiel de truitelles est probablement très faible. De plus, ces truites déversées peuvent avoir participé à la reproduction pour la première fois pendant l'hiver 1992-1993 et les juvéniles de l'année $(0+)$ sont trop petits pour être pris en compte dans ce prélèvement (longueur minimale $11 \mathrm{~cm}$ ).

Par contre, les truitelles déversées sur la partie amont du bassin au printemps 1991 ou 1992 auraient dû, après dévalaison, être capturées lors de ce prélèvement. Or, aucun individu de pisciculture n'a été identifié dans cet échantillon.

\section{CONCLUSION}

Cette étude montre la part importante prise par les populations de truites sauvages dans la reconstitution des populations après une perturbation majeure du milieu liée à une crue de grande amplitude. La mise en réserve du bassin permet la protection des géniteurs ayant échappé à la crue et favorise ainsi une recolonisation plus rapide du milieu.

Parallèlement aux capacités naturelles du milieu à se régénérer, la disparition apparente des sujets d'origine domestique et le faible degré d'introgression des souches sauvages constaté, ne plaident pas en faveur des déversements de truitelles pour repeupler les rivières, même après ce type d'événements catastrophiques.

En perspective, il serait cependant intéressant de pouvoir vérifier l'apport des individus de pisciculture à la reproduction, en caractérisant au niveau génétique les juvéniles de l'année. De plus, une étude comparative incluant plusieurs bassins permettrait d'intégrer l'influence des caractéristiques environnementales, afin de mieux cerner les processus et de pouvoir les généraliser, ce type de perturbations n'étant pas inféodé à la Corse mais pouvant intervenir sur l'ensemble du bassin méditerranéen.

\section{REMERCIEMENTS}

Le suivi démographique et l'étude génétique ont été réalisés sous maîtrises d'ouvrage respectives de la Fédération Interdépartementale des A.P.P.M.A. et du Parc Naturel Régional de Corse, dans le cadre du Xème Contrat de Plan avec l'aide financière de l'Etat et de la Collectivité Territoriale de Corse. Nos remerciements vont à la brigade des Gardes-Pêche de la Fédération Interdépartementale qui ont participé aux échantillonnages.

\section{BIBLIOGRAPHIE}

BARIL D., LE BARON M., 1993. Suivi piscicole de l'Abatesco suite à la crue de septembre 1989. Conseil Supérieur de la Pêche, Délégation Régionale $n^{\circ} 8,23 p+$ annexes.

BERREBI P., 1994. Etude génétique des truites de Corse. Rapport final 1994 - commande 1993 du Parc Naturel Régional de Corse, $16 p+$ annexes.

CARLE F.L., STRUB M.R., 1978. A new method for estimating population size from removal data. Biometrics, 34, 621-630.

D.D.E. (Direction Départementale de l'Equipement de Haute-Corse, SOGREAH Grenoble), 1990-a. Amélioration des écoulements en crue au franchissement du Fium'Orbo Protection des appuis de l'ouvrage principal.

D.D.E. (Direction Départementale de l'Equipement de Haute-Corse, SOGREAH Grenoble), 1990-b. Confortement et protection des appuis de l'ouvrage de franchissement du Travo.

D.D.E. (Direction Départementale de l'Equipement), 1994. Commune de Prunelli di Fium'Orbo - Etude indicative de risque d'inondation - Notice hydrologique et hydraulique. SAFEGE, CODRA. 
DIREN Corse (Direction Régionale de l'Environnement), SEMA (Service de l'Eau et des Milieux Aquatiques), Ministère de l'Environnement, 1993. Note préliminaire concernant le débit de référence introduit par le décret "nomenclature» du 29 mars 1993, en application de l'article 10 de la loi sur l'eau du 3 janvier 1992, 11 p.

FÉDÉRATION DES AAPP (Fédération Interdépartementale des Associations Agréées de Pêche et de Pisciculture de la Corse, Service Régional d'Aménagement des Eaux, Direction Départementale de l'Agriculture et de la Forêt), 1991. Schéma de vocation piscicole et halieutique de la Haute-Corse - Rapport de synthèse, $22 \mathrm{p}$.

GUYOMARD R., 1989-a. Gestion génétique des populations naturelles : l'exemple de la truite commune. Bull. Fr. Pêche Piscic., 314, 136-145.

GUYOMARD R., 1989-b. Diversité génétique de la truite commune. Bull. Fr. Pêche Piscic., 314, 118-135.

GUYOMARD R., KRIEG F., 1986. Mise en évidence d'un flux génétique entre populations naturelles de truite fario et souche de repeuplement dans deux rivières de Corse. Bull. Fr. Pêche Piscic., 303, 134-140.

KRIEG F., 1984. Recherche d'une différenciation génétique entre populations de Salmo trutta. Thèse 3ème cycle, Univ. Paris Sud (Orsay), $92 \mathrm{p}$.

MAISSE G., BAGLINIĖRE J.L., 1991. Biologie de la truite commune (Salmo trutta L.) dans les rivières françaises. In La truite, biologie et écologie. Eds J.L. Baglinière et $G$. Maisse, INRA / ENSA, 304 p.

PASTEUR N., PASTEUR G., BONHOMME F., CATALAN J., BRITTON-DAVIDIAN J., 1987. Manuel de génétique par électrophorèse des protéines. Collection technique et documentation, Lavoisier Paris, $217 \mathrm{p}$.

ROCHÉ B., 1987. Poissons dulçaquicoles de la Corse : étude du peuplement piscicole des eaux courantes et des populations de truites. Service Régional de l'Aménagement des Eaux de la Corse, 40 p. 